\title{
The impact of population-based faecal occult blood test screening on colorectal cancer mortality: a matched cohort study
}

\author{
G Libby ${ }^{*}$, , DH Brewster ${ }^{2}$, PL McClements ${ }^{2}$, FA Carey ${ }^{3}$, RJ Black$^{2}$, J Birrell ${ }^{2}$, CG Fraser' and RJC Steele ${ }^{1,4}$ \\ 'Scottish Bowel Screening Research Unit, Kings Cross, Clepington Road, Dundee DD3 8EA, UK; ${ }^{2}$ National Services Division, NHS National Services \\ Scotland, I South Gyle Crescent, Edinburgh EHI 2 9EB, UK; ${ }^{3}$ Department of Pathology, Ninewells Hospital and Medical School, Dundee DDI 9SY, UK; \\ ${ }^{4}$ Department of Surgery and Molecular Oncology, Ninewells Hospital and Medical School, Dundee DDI 9SY, UK
}

\begin{abstract}
BACKGROUND: Randomised trials show reduced colorectal cancer (CRC) mortality with faecal occult blood testing (FOBT). This outcome is now examined in a routine, population-based, screening programme.

METHODS: Three biennial rounds of the UK CRC screening pilot were completed in Scotland (2000-2007) before the roll out of a national programme. All residents (50-69 years) in the three pilot Health Boards were invited for screening. They received a FOBT test by post to complete at home and return for analysis. Positive tests were followed up with colonoscopy. Controls, selected from non-pilot Health Boards, were matched by age, gender, and deprivation and assigned the invitation date of matched invitee. Follow-up was from invitation date to 31 December 2009 or date of death if earlier.

RESULTS: There were 379655 people in each group (median age 55.6 years, $51.6 \%$ male). Participation was 60.6\%. There were $96 \mathrm{I}(0.25 \%)$ CRC deaths in invitees, I 056 (0.28\%) in controls, rate ratio (RR) 0.90 (95\% confidence interval (Cl) 0.83-0.99) overall and 0.73 (95\% Cl 0.65-0.82) for participants. Non-participants had increased CRC mortality compared with controls, RR I.2I (95\% Cl 1.06-1.38).

CONCLUSION: There was a $10 \%$ relative reduction in CRC mortality in a routine screening programme, rising to $27 \%$ in participants. British Journal of Cancer (2012) I 07, 255-259. doi:I0.1038/bjc.20 I2.277 www.bjcancer.com
\end{abstract}

Published online 26 June 2012

(c) 2012 Cancer Research UK

Keywords: faecal occult blood testing; colorectal cancer mortality

A Cochrane review of the four randomised controlled trials of screening for colorectal cancer (CRC) using faecal occult blood testing (FOBT) has shown a reduction in cause-specific mortality, with an intention to screen analysis, of $16 \%$, rising to $23 \%$ when adjusted for compliance (Towler et al, 1998). On the basis of these results a demonstration pilot of population-based FOBT was commissioned in the United Kingdom and the successful completion has in turn led to a roll out of programmes in all four UK countries.

Process measures from both the Scottish and English arms of the UK pilot have been quantified and published (Steele et al, 2009; Moss et al, 2012). These include cancer detection rate, stage distribution, and positive predictive value. Disease-specific mortality is the most important outcome measure, and the main aim of this study was to examine whether the reduction in CRC mortality seen in the randomised controlled trials could be matched at the population level in practice.

It is not certain that the reduction in CRC mortality can be achieved as randomised controlled trials are underpinned by high levels of motivation within a restricted team and some aspects of the published trials, for example, diet restriction (Mandel et al, 1993; Kewenter et al, 1994; Kronborg et al, 1996) and high rates of colonoscopy (Mandel et al, 1993) would not be feasible in routine population screening. One non-randomised study has been

*Correspondence: G Libby; E-mail: g.libby@dundee.ac.uk Received I4 March 2012; revised 27 May 2012; accepted 29 May 20 I2; published online 26 June 2012 carried out in France (Faivre et al, 2004) but the screened and non-screened groups were matched at the level of administrative area and no account was taken of socio-economic deprivation, which is a key factor in screening uptake (Steele et al, 2010).

Therefore, a retrospective cohort study was carried out to compare CRC mortality in the general population routinely invited for FOBT screening with people not invited but matched at the individual level for gender, year of birth, and socio-economic status.

\section{MATERIALS AND METHODS}

\section{Design of study}

The design of the Scottish arm of the UK pilot of FOBT screening provided the opportunity to compare individuals resident in the three National Health Service (NHS) boards selected to be involved in the screening pilot with a matched group drawn from the remaining NHS boards.

The pilot in Scotland has been described elsewhere (Steele et al, 2009) but in brief individuals aged 50-69 years and living in one of the three NHS board areas were sent a FOBT kit (hema-screen, Immunostics, Ocean, NJ, USA) by post to complete at home and then return to the central laboratory for analysis. This was a traditional style test consisting of six windows of filter paper impregnated with guaiac gum. Participants with 5 or 6 positive windows were offered colonoscopy. Those with 1-4 windows positive were asked to complete a further guaiac test and colonoscopy was offered to those with any one window positive. 
There were no dietary restrictions on the initial test. The pilot consisted of three rounds of biennial screening carried out between 2000 and 2007 (29 March 2000-31 December 2002, 2 December 2002-30 April 2005, and 6 May 2005-20 July 2007).

\section{Study population}

The study population and associated dataset were assembled by the Information Services Division of NHS National Services Scotland. All invitees for screening had a community health index number that is a unique patient identifier for all individuals registered with a general practitioner in Scotland. A list of the community health index numbers of those invited in at least one of the three rounds of screening was compiled and a unique control person for each invitee, matched for gender, year of birth, and quintile of deprivation as defined from the Scottish Index of Multiple Deprivation based on postcode of residence was identified (www.scotland.gov.uk/topics/Statistics/SIMD). Controls were selected from NHS boards with background mortality for CRC comparable to that in the pilot NHS boards. Male controls were drawn from nine NHS boards and females from five NHS boards. Controls were not selected from one NHS board because some of the residents had been invited for screening previously as part of a UK randomised controlled trial of flexible sigmoidoscopy.

Each control was assigned the date of first screening invitation of their matched invitee which was used as a proxy screening date. The invitee and matched control in each pair were then followed from this date to 31 December 2009 or date of death if earlier. Using the community health index number, the study population was record linked to a number of national databases to compile the data required for the study. Colorectal cancer diagnoses were obtained from the Scottish Cancer Registry which since 1958 has collected data on all new cases of cancer arising in the Scottish population. Hospital admissions for CRC were identified from the Scottish Morbidity Record database and the date and cause of death were identified from the National Records of Scotland database. For this study, only the underlying cause of death was used and was classified as CRC if coded as ICD10 C18-C20. No account was taken of potential migration from Scotland and individuals were deemed to be alive at 31 December 2009 if no date of death was recorded in the National Records. The invited group was additionally record linked to the screening database to identify participation and screening test results.

\section{Exclusions}

All individuals in the appropriate age range, resident in the pilot NHS boards and who had a community health index number were eligible to be invited for FOBT screening. Before the first round of screening, however, general practitioners were given the opportunity to identify any patients for whom an invitation for screening was considered to be inappropriate, for example, patients who were terminally ill. This was done through the use of prior notification lists. Their use was discontinued after the first pilot screening round.

Once the study population had been compiled, matched pairs were excluded if either person had had a hospital admission for or a diagnosis of CRC before the screening date or if the control person had died before the screening date.

\section{Statistical analysis}

There were no formal calculations of sample size. All individuals invited as part of the pilot were included at the start of the study with the initial aim to find a unique matched control for each.

To compare the results from this study with those from the randomised controlled trials, an 'intention to screen' approach was used for the main analyses with secondary analyses adjusted for participation. This was defined as the return of at least one screening test offered. Demographic differences between participants and non-participants are regularly seen that could indicate different underlying risks. Therefore, in addition, the rate of CRC mortality in those accepting screening was compared with the controls using the method proposed by Cuzick et al (1997) for randomised controlled trials that potentially takes account of these differences.

The primary outcome measure was CRC mortality. Person years of follow-up in each group were calculated from the screening/ matched date to 31 December 2009 or date of death if earlier. Cumulative CRC mortality rates with $95 \%$ CIs were plotted by year from the screening/matched date for the invited and control groups. These were calculated by the Nelson-Aalen method to take account of length of follow-up (Aalen, 1978).

Overall CRC mortality rates in each group were calculated as the number of deaths divided by the person years of follow-up. Rate ratios (RRs) were calculated using the Mantel Haenzel method to compare these rates in the invited and control groups overall and by age groups (50-59 and 60-69 years), gender, and participation.

The number needed to be screened was calculated as proposed by Tabar et al (2004) to account for the rate of screening compliance.

All analyses were carried out with STATA (version 10.0, StataCorp, College Station, TX, USA).

\section{RESULTS}

There were 410320 individuals invited for screening in at least one of the three rounds of the pilot. A unique matched control could not be found for 19232 of whom $91 \%$ were female and $87 \%$ were in the two least deprived quintiles. There were 4824 matched pairs excluded where a control had died before the matched screening date or a control or invited person had been diagnosed with or had a hospital admission for CRC before the screening/matched date.

The number of deaths from all causes over the period of screening round one (Figure 1) were plotted and appeared to show an under-representation of deaths in the group invited for screening. This pattern was not seen in rounds two and three and might suggest an effect of the prior notification lists sent to general practitioners. A decision was therefore made to exclude all individuals who had died before 1 February 2002 and their matched invited or control person (6609 pairs). This date was chosen arbitrarily since after this, there was $<20 \%$ difference between the numbers of deaths in the two groups. Among the exclusions were 118 individuals who had died of CRC, 59 in each group. These exclusions left 379655 individuals in each of the invited and control groups (Figure 2).

The demographic characteristics of each group are shown in Table 1. The unequal distribution of the quintiles of deprivation reflects the selection of invited and control groups from a restricted number of NHS boards. In round one, the 50-54 years age group accounted for $33.1 \%$ of the study population but this rose to $47.4 \%$ for the study as a whole due to the majority of those invited for the first time in rounds two and three being aged 50 or 51 years thus increasing the proportion in the youngest age group. All cause mortality in the study population was $8.1 \%$. In the invited group, there were no deaths related to the screening colonoscopy itself. There were $961(0.25 \%)$ and $1056(0.28 \%)$ deaths from CRC in the invited and control groups respectively $(P=0.03$ chi-squared test). A 'maximum' result for those in the invited group was defined over all potential rounds and ranged from 'not provided' (a test kit not being returned) to 'positive'. Overall $60.6 \%$ of the invited group had returned at least one screening test.

Figure 3 shows the overall cumulative mortality from CRC in both groups with lower mortality in the invited group appearing around 4 years after screening. Cumulative mortality rates in the participants and non-participants compared with their respective 


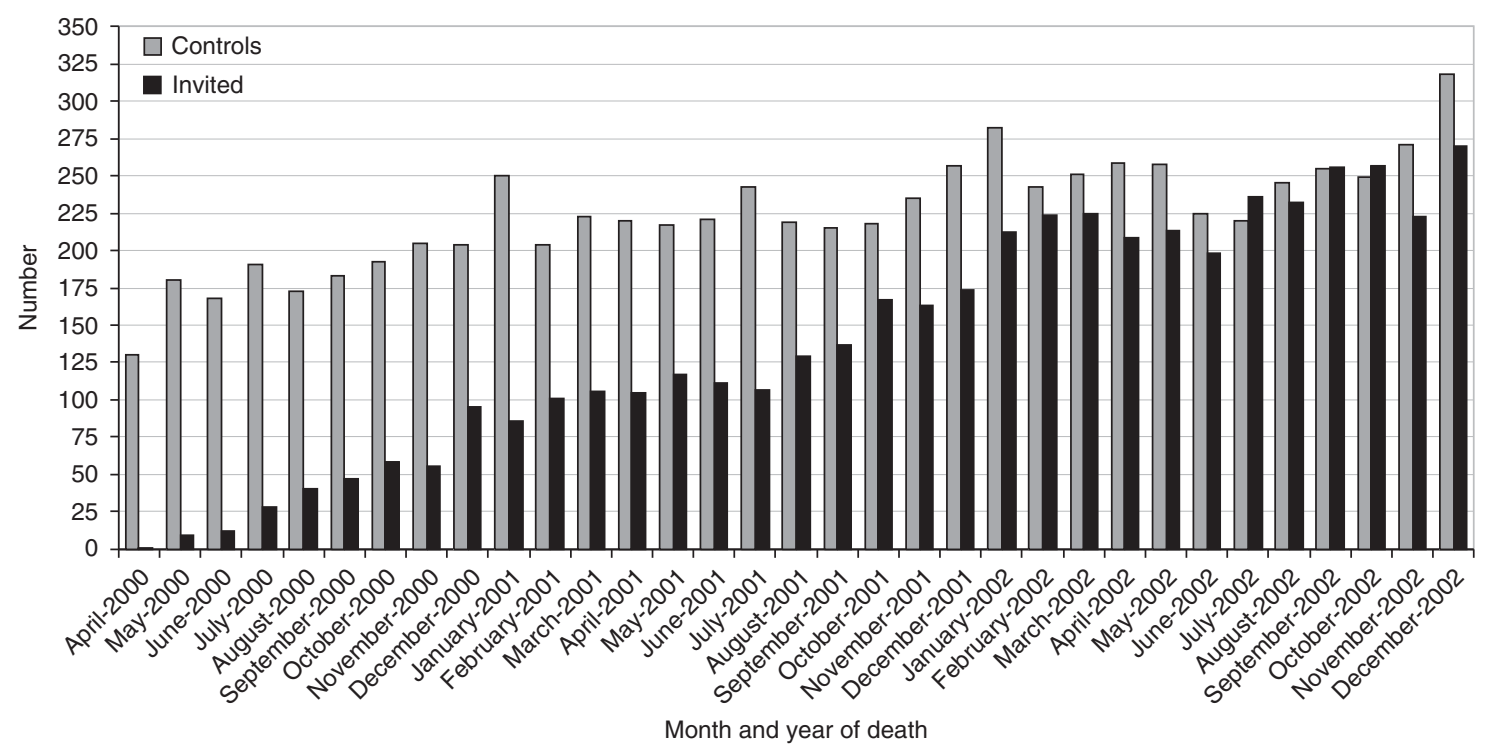

Figure I Number of all cause deaths in invited for screening and control groups.

Pilot NHS boards Invited

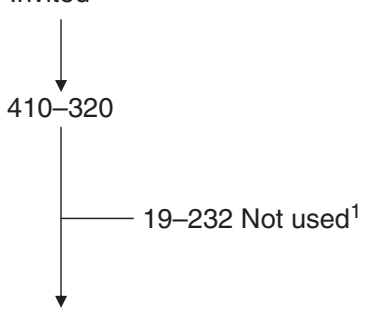

391-088

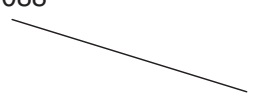

4824 Matched pairs excluded ${ }^{2}$

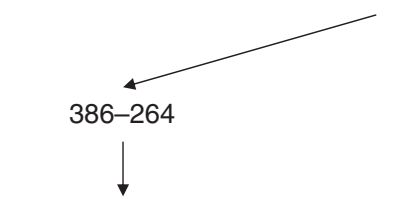

2061 Died

$<1$ February 2002

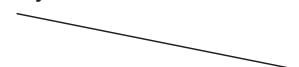

6609 Matched pairs excluded ${ }^{3}$ (2061+4635-4687 common to both groups)
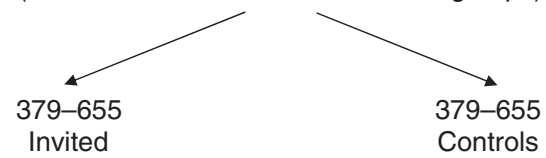

${ }^{1}$ No matched control available.

${ }^{2}$ Died before screening/matched date or had colorectal cancer diagnosed before screening/matched date.

${ }^{3}$ To account for effect of prior notification lists.

Figure 2 Derivation of study population.
Non pilot NHS boards Matched controls

379-655

4635 Died $<1$ February 2002

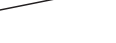




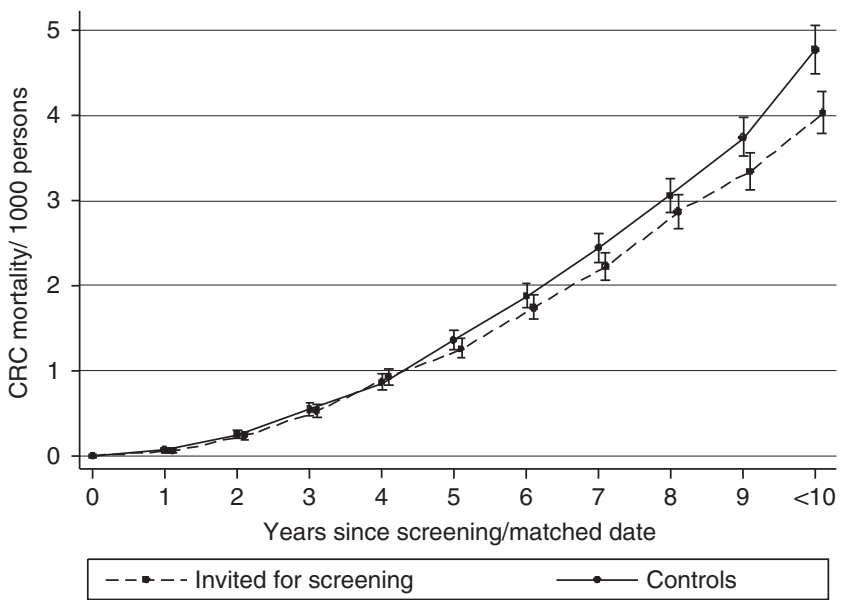

Figure 3 Cumulative mortality from colorectal cancer. Rate and $95 \% \mathrm{Cl}$ (Nelson-Aalen estimates).

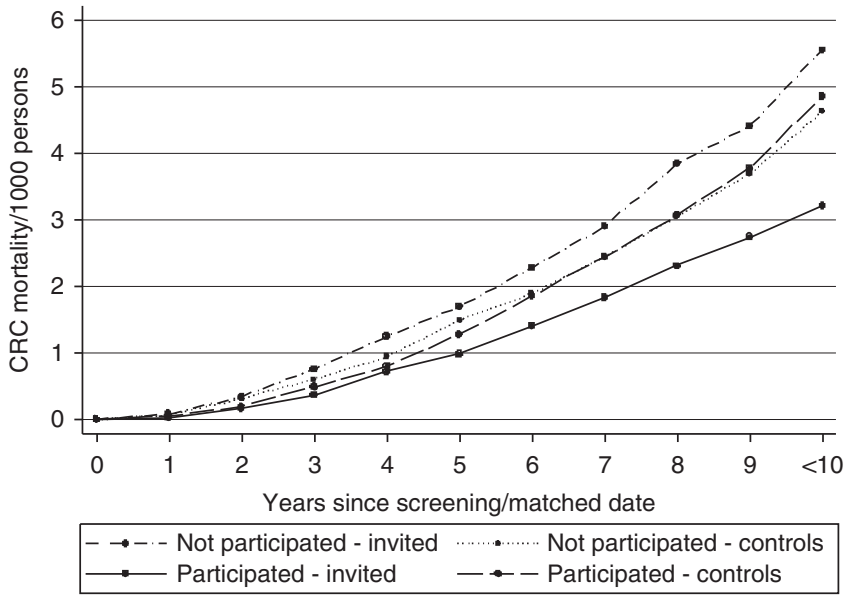

Figure 4 Cumulative mortality from colorectal cancer by participation (Nelson-Aalen estimates).

control groups are shown in Figure 4. As seen elsewhere (Scholefield et al, 2002), CRC mortality rates were lower in the participants compared with the non-participants and this difference increased with time following screening.

The overall CRC mortality rate/1000 person years was $0.35(95 \%$ CI $0.33-0.39$ ) for the invited group and 0.38 (95\% CI 0.36-0.41) for the controls. The RRs showed a $10 \%$ relative reduction with intention to screen $\mathrm{RR}=0.90$ (95\% CI $0.83-0.99)$ and a $27 \%$ relative reduction in participants compared with their matched controls $\mathrm{RR}=0.73$ (95\% CI 0.65-0.82). The Cuzick method showed a reduction of $17 \% \mathrm{RR}=0.83(95 \% \mathrm{CI} 0.79-0.87)$. There was also a significant reduction in CRC mortality with invitation for screening for those aged 50-59 years and for males (Table 2).

A sensitivity analysis was carried out to examine the effect on the overall RR of excluding all those who died before 1 February 2002 and their matched person. The RR remained unchanged (0.90, 95\% CI 0.83-0.99) implying no effect.

The number needed to screen was calculated using data from round 1 to allow the maximum time of follow-up. There were 155496 participants in round 1 with 893 CRC deaths in the invited group and 987 in the controls. The estimated number of CRC deaths prevented was 94 (987-893). The number of people needed to be screened for a period of 7 years to prevent one death from CRC over a follow-up period of 9 years was therefore estimated to
Table 2 Rate ratios of CRC mortality comparing the invited and control groups

\begin{tabular}{|c|c|c|c|}
\hline & \multicolumn{2}{|c|}{ Deaths from CRC } & \multirow[b]{2}{*}{$\begin{array}{l}\text { Rate ratio } \\
(95 \% \mathrm{Cl})\end{array}$} \\
\hline & $\begin{array}{l}\text { Invited } \\
N(\%)\end{array}$ & $\begin{array}{l}\text { Controls } \\
N(\%)\end{array}$ & \\
\hline All & $96 \mid(0.25)$ & $1056(0.28)$ & $0.90(0.83-0.99)$ \\
\hline Person years & 2777086 & 2767873 & \\
\hline Participants & $494(0.21)$ & $664(0.29)$ & $0.73(0.65-0.82)$ \\
\hline Participants-Cuzick method & & & $0.83(0.79-0.87)$ \\
\hline Non-participants & $467(0.31)$ & $392(0.26)$ & $1.21(1.06-1.38)$ \\
\hline \multicolumn{4}{|c|}{ Age group at screening date (years) } \\
\hline $50-59$ & $345(0.14)$ & $401(0.16)$ & $0.86(0.74-0.99)$ \\
\hline $60-69$ & $616(0.49)$ & $655(0.52)$ & $0.94(0.84-1.05)$ \\
\hline Females & $375(0.20)$ & $397(0.22)$ & $0.94(0.82-1.09)$ \\
\hline Males & $586(0.29)$ & $659(0.34)$ & $0.89(0.79-0.99)$ \\
\hline
\end{tabular}

Abbreviation: $\mathrm{CRC}=$ colorectal cancer.

be 1654 (155 496/94). Of 1654 individuals screened over this time period, $33(2 \%)$ could be expected to have a positive test and 28 $(85.5 \%$; Steele et al, 2009) to have a colonoscopy.

\section{DISCUSSION}

This study reports the impact of FOBT screening on CRC mortality in a routine, population-based programme serving a true general population. The results show that such screening reduces diseasespecific mortality by $10 \%$ rising to $27 \%$ when adjusted for participation.

A particular strength of the study was the assessment of CRC mortality over the same time period in a population being invited for screening for the first time and a matched cohort with no history of screening. A limitation, however, was the lack of a unique control for 19232 persons invited for screening due to restricting the selection of matched controls to those from NHS boards with comparable background mortality. It is not known what effect if any this would have had on the results but due to the large size of the study population this group accounted for only $5 \%$ of those invited for screening.

The reduction in mortality was lower than that seen in the metaanalysis, which was $16 \%$ though the individual trial results ranged from 12 to 19\% (Towler et al, 1998). The reason for this is not clear, but there were two important differences in our study compared with the randomised controlled trials. First, the study population was much younger with $47 \%$ aged between 50 and 54 years and only $33 \%$ aged 60 years or older. This compares to between 50 and $100 \%$ aged 60 years or over in the four trial populations. Consequently, this study had substantially lower all cause mortality $(8.1 \%)$ compared with the trials (16.6-30.5\%). In contrast, CRC as the cause of death accounted for $3.3 \%$ of all deaths in the control group, which was similar to that in the trials (3.0-3.6\%). Colorectal cancer incidence and mortality increase with age and the lower age range and associated lower risk in the study population may have reduced the benefit from screening.

The second difference was that the cause of death was defined only using information from death certificates. In contrast, all the trials used an expert committee that examined the death certificate information along with relevant medical records to define the underlying cause of death. In the Nottingham trial (Hardcastle et al, 1996), deaths from CRC were classified in two ways, 'certified' from death certificate information only and 'verified' after additional case note review. The latter approach resulted in more deaths being defined with a cause of CRC. There was a 
statistically significant reduction in CRC mortality of $15 \%$ only in the larger 'verified' group. The 'certified' group showed a nonsignificant reduction of $12 \%$.

These two differences may have contributed to the lower reduction in mortality seen in this study compared with the metaanalysis (Towler et al, 1998). In addition, colonoscopy was offered directly to those with 5 or 6 FOBT windows positive (strong positive) but not those with 1-4 windows positive (weak positive) until they had another positive FOBT result. This approach was based on the algorithm used in the Nottingham trial and was designed, considering the available colonoscopy resource, to achieve a positivity rate of $2 \%$. It has been suggested that this is too conservative an approach resulting in a diminution of the benefit of screening compared with the benefit if all those with a positive test result were referred for colonoscopy. Another trial in the United States gained an increased positivity rate through rehydration of the FOBT (Mandel et al, 1993). This resulted in higher colonoscopy rates and showed an increased reduction in mortality of $19 \%$ but with a lower positive predictive value because of the increased number of false positive test results. Offering colonoscopy directly to weak positives, therefore, may have led to a greater reduction in mortality, but a recent study has shown that where colonoscopy is undertaken following a weak positive test result, the positive predictive value for cancer is $8.8 \%$ compared with $26 \%$ for those with a strong positive test (Lee et al, 2011). It is encouraging, however, that a significant reduction in CRC mortality was achieved despite the limitations that are important factors in routine, real-world screening programmes in contrast to studies undertaken with the advantages of clinical trial support.

It was found that all cause mortality was slightly greater in the control group than in the invited group raising the possibility that the reduction in CRC mortality could have been partly due to differences in overall mortality. However, the statistically significant differences between the groups were confined to non-

\section{REFERENCES}

Aalen OO (1978) Non-parametric inference for a family of counting processes. Ann Statis 6: 701-726

Cuzick J, Edwards R, Segnan N (1997) Adjusting for non-compliance and contamination in randomised clinical trials. Stat Med 16: 1017-1029

Faivre J, Dancourt V, Lejeune C, Tuzi MA, Lamour J, Gerard D, Dassonville F, Bonithon-Kopp C (2004) Reduction in colorectal cancer mortality by fecal occult blood screening in a French controlled study. Gastroenterology 126: $1674-1680$

Hardcastle JD, Chamberlain JO, Robinson MHE, Moss SM, Amar SS, Balfour TW, James PD, Mangham CM (1996) Randomised controlled trial of faecal-occult-blood screening for colorectal cancer. Lancet 348: 1472-1477

Kewenter J, Brevinge H, Engaras B, Haglind E, Ahran C (1994) Results of screening, rescreening and follow-up in a prospective randomised study for detection of colorectal cancer by fecal occult blood testing. Scand $J$ Gastroenterol 29: 468-473

Kronborg O, Fenger C, Olsen J, Jorgensen OD, Sondergaard O (1996) Randomised study of screening for colorectal cancer with faecal-occult blood test. Lancet 348: 1467-1471

Lee TJW, Clifford GM, Rajasekhar P, Rutter MD, Kometa S, Ritchie MC, Waddup G, Nylander D, McNally RJ, Rees CJ (2011) High yield of colorectal neoplasia detected by colonoscopy following a positive faecal occult blood test in the NHS Bowel Cancer Screening Programme. J Med Screen 18: 82-86

Mandel JS, Bond JH, Church TR, Snover DC, Bradley GM, Schuman LM, Ederer F, For the Minnesota Colon Cancer Control Study (1993) Reducing mortality from colorectal cancer by screening for fecal occult blood. N Engl J Med 328(19): 1365-1371 cancer causes of death with no differences between the groups for any cancer other than CRC (data not shown). Thus, it is unlikely that the reduction in CRC mortality could be due to background differences in overall mortality.

Previously published studies of routine screening programmes have shown that screening with FOBT leads to an increased detection of CRC at early stages of disease (Steele et al, 2009; Moss et al, 2012). This study shows that these outcomes translate into a reduction in cause-specific mortality justifying the introduction of routine population-based screening. It seems likely that a greater impact of screening on CRC mortality would be realised with increased participation.

\section{ACKNOWLEDGEMENTS}

We thank Chris Povey (NHS National Services Scotland) for his work in identifying the control population. This work was supported by a grant from the Chief Scientist Office (Grant No $\mathrm{CZH} / 6 / 4)$, Scottish Government Health Directorates to establish a bowel screening research unit.

\section{Ethical approval}

Ethical approval was not sought for the demonstration pilot. This was a decision made by the National Screening Committee and endorsed by the United Kingdom Departments of Health on the grounds that screening for colorectal cancer using FOBT is of proved efficacy and the pilot constituted an evaluation of the feasibility of introducing a screening programme into the NHS. Permission to access and analyse the anonymised-linked data presented in this paper was granted by the Community Health Index Advisory Board and the Privacy Advisory Committee, National Services Scotland.

Moss SM, Campbell C, Melia J, Coleman D, Smith S, Parker R, Ramsell P, Patnick J, Weller DP (2012) Performance measures in three rounds of the English bowel cancer screening pilot. Gut 61: 101-107

Scholefield JH, Moss S, Sufi F, Mangham CM, Hardcastle JD (2002) Effect of faecal occult blood screening on mortality from colorectal cancer: results from a randomised controlled trial. Gut 50(6): $840-844$

Steele RJ, Kostourou I, McClements P, Watling C, Libby G, Weller D, Brewster DH, Black R, Carey FA, Fraser C (2010) Effect of gender, age and deprivation on key performance indicators in a FOBT based colorectal screening programme. J Med Screen 17(2): 68-74

Steele RJ, McClements PL, Libby G, Black R, Morton C, Birrell J, Mowat NA, Wilson JA, Kenicer M, Carey FA, Fraser CG (2009) Results from the first three rounds of the Scottish demonstration pilot of FOBT screening for colorectal cancer. Gut 58(4): 530-535

Tabar L, Vitak B, Yen MF, Chen HH, Smith RA, Duffy SW (2004) Number needed to screen: lives saved over 20 years of follow-up in mammographic screening. J Med Screen 11: 126-129

Towler B, Irwig L, Blasziou P, Kewenter J, Weller D, Silagy C (1998) A systematic review of the effects of screening for colorectal cancer using the faecal occult blood test, Hemoccult. BMJ 317(7158): 559-565

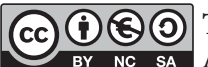

This work is licensed under the Creative Commons Attribution-NonCommercial-Share Alike 3.0 Unported License. To view a copy of this license, visit http://creativecommons. org/licenses/by-nc-sa/3.0/ 